Revue d'histoire de l'Amérique française

REVUE D.HISTOIRE DE L'AMÉRIQUE FRANÇAISE

\title{
L’agriculture de la Mauricie et du Québec, 1850-1950
}

\section{Normand Séguin}

Volume 35, numéro 4, mars 1982

URI : https://id.erudit.org/iderudit/304011ar

DOI : https://doi.org/10.7202/304011ar

Aller au sommaire du numéro

Éditeur(s)

Institut d'histoire de l'Amérique française

ISSN

0035-2357 (imprimé)

1492-1383 (numérique)

Découvrir la revue

Citer cet article

Séguin, N. (1982). L'agriculture de la Mauricie et du Québec, 1850-1950. Revue d'histoire de l'Amérique française, 35(4), 537-562.

https://doi.org/10.7202/304011ar d'utilisation que vous pouvez consulter en ligne.

https://apropos.erudit.org/fr/usagers/politique-dutilisation/ 


\title{
L'AGRICULTURE DE LA MAURICIE ET DU QUÉBEC 1850-1950*
}

\author{
NORMAND SÉGUIN \\ Département des sciences humaines \\ Université du Québec à Trois-Rivières
}

On l'a suffisamment répété, nous connaissons encore mal les transformations de l'agriculture québécoise contemporaine. Fautil préciser que nous connaissons encore moins bien l'évolution historique des agricultures régionales et québécoises.

C'est par à-coups, par segments, que les traits généraux de la production agricole du Québec et de ses régions se laissent appréhender à travers des écrits nombreux, mais aux vertus inégales ${ }^{1}$. Cette méconnaissance des faits bruts, dans leur contexte et leur temporalité spécifiques, gêne notre compréhension du monde rural en général et de la paysannerie en particulier. Ce texte est une tentative de surmonter - pour une région en particulier, la Mauricie - le sérieux handicap que représente l'absence d'un «référentiel» suffisamment indicatif des traits fondamentaux de l'agriculture en période contemporaine.

Deux mouvements de fond ont marqué profondément l'agriculture québécoise durant la seconde moitié du 19e siècle. D'un côté on assiste à un début d'intégration de la production agricole à l'économie de marché; ce processus pousse le paysan vers de nouvelles orientations - la production du lait en particulier autour de 1870 — , l'incite au relèvement de la productivité de son labeur, bref l'arrache lentement à l'autosuffisance et l'élève à un premier niveau de commercialisation. D'un autre côté, on assiste à l'extension de l'oekoumène vers l'intérieur et les zones périphériques; c'est le domaine d'une agriculture marginale dont l'existence est en fonction étroite des rapports que les paysans entretiennent par leur travail avec des activités extra-agricoles, forestières principalement.

* Ce texte s'inscrit dans les travaux du Groupe de recherche sur la Mauricie. Depuis sa formation, celui-ci a reçu un appui financier des organismes suivants: l'Université du Québec à Trois-Rivières, la Société Saint-Jean-Baptiste de la Mauricie, le Ministère de l'Éducation du Québec, le Conseil de recherches en sciences humaines du Canada. Nous tenons à remercier Louise Verreault-Roy et Alain Ledoux qui ont longuement collaboré au traitement des données afférentes à cette publication. Nous disons aussi notre gratitude à René Hardy et Jean-Claude Robert qui ont aidé par leurs commentaires à améliorer la présentation de cette recherche.

Pour un survol des travaux consacrés à la question agricole au Québec, voir Normand Séguin, L'agriculture et la colonisation au Québec (Montréal, Boréal Express, 1980), 12-26. 
Entraînée par l'industrialisation et l'urbanisation, l'agriculture québécoise poursuit, au 20e siècle, son intégration à l'économie de marché et atteint des niveaux de plus en plus élevés de commercialisation, tant et si bien qu'entrée dans l'ère du complexe agro-industriel au cours des années 1950, elle est quasi entièrement soumise à l'économie industrielle et urbaine. Le paysan a perdu à toutes fins utiles son autonomie de petit producteur. L'agriculture ne fonctionne plus alors que comme «un» secteur de l'économie globale. Des équilibres anciens s'effondrent, - c'est la désarticulation, par exemple, du système agro-forestier établie au 19e siècle - entraînant l'effondrement démographique et la «désertification» dans de nombreuses zones. Le cas mauricien offre l'exemple d'un espace agraire qui reproduit ces grandes tendances qui ont fortement déterminé le développement différentiel de l'agriculture québécoise.

La Mauricie, comme entité régionale, est un phénomène relativement récent. En effet, jusqu'aux premières décennies du 19e siècle, les basses terres occupées de la région actuelle (en gros, les terres seigneuriales en bordure du fleuve) apparaissent comme un simple segment du continuum reliant Québec et Montréal. TroisRivières y tient tout juste le rôle d'un petit centre de services et de transit à la dimension d'un espace rural environnant encore très peu déployé vers l'intérieur. Le début de l'exploitation forestière, le long de la rivière du Loup vers 1825 et de la rivière Batiscan vers 1835 , et son démarrage en force le long de la rivière Saint-Maurice au cours des années 1850, modifient radicalement cet état de fait. Agissant comme activité motrice, l'exploitation forestière de la seconde moitié du 19e siècle structure une économie préindustrielle de type agro-forestier, le Saint-Maurice jouant le rôle d'axe majeur et de principe intégrateur d'un nouvel espace régional. Trois-Rivières en est le lieu principal et l'unique centre urbain. Le démarrage de l'industrie à la fin du $19 \mathrm{e}$ siècle et son essor pendant les premières décennies du 20e siècle, modifient en profondeur la morphologie de cet espace régional en jetant les bases de l'actuel réseau urbain et en accélérant la mutation du domaine agricole.

Notre propos n'est pas ici de faire une analyse globale du phénomène complexe qu'est la production agricole en Mauricie. Le Groupe de recherche sur la Mauricie s'y emploie depuis près de quatre ans déjà ${ }^{2}$ et il devra poursuivre ses recherches pendant quel-

2 Le Groupe de recherche sur la Mauricie, sous les signatures de Normand Séguin, René Hardy et Louise Verreault-Roy, a déjà fait paraître L'agriculture en Mauricie, dossier statistique: 1850-1950. Cahier no 2, U.Q.T.R., 1979, 175 p. Ce cahier présente une mise en séries des principales données de l'agriculture mauricienne; l'introduction comporte de nombreuses notes méthodologiques sur l'utilisation des recensements dans l'étude de l'évolution de l'agriculture. 
ques années encore avant de pouvoir proposer une lecture satisfaisante de l'évolution de l'agriculture et de la paysannerie en Mauricie. Nous voulons simplement, dans ce texte, cerner certains grands paramètres de l'espace agricole à l'aide d'indicateurs confectionnés à partir des données publiées des recensements fédéraux. Nous nous arrêtons à l'année 1951, qui marque grosso modo la fin de la période d'histoire rurale que le Groupe a choisi d'étudier, laissant à d'autres le soin de scruter les dernières décennies.

La démarche comporte deux objectifs principaux: situer l'agriculture régionale dans l'ensemble québécois et caractériser son évolution différentielle dans les trois comtés présentés ici comme entité régionale. Cette première évaluation de l'agriculture mauricienne reste partielle puisqu'elle ne tient compte que des données relatives aux occupants, aux superficies, aux grandes productions végétales et animales, et aux grands élevages. Nous avons en chantier une étude plus globale de la production agricole portant cette fois sur trois paroisses représentatives des étapes historiques de la formation de l'espace régional: une paroisse du vieux terroir seigneurial en bordure du fleuve (Champlain), particulièrement exposée à l'influence du marché; une paroisse du premier front pionnier (Saint-Stanislas), à mi-chemin entre le fleuve et la barrière du socle laurentien; enfin, une paroisse du dernier front pionnier adossé aux Laurentides (Saint-Tite), témoin des chambardements survenus sur les marges de l'oekoumène. La présente analyse est davantage centrée sur les grands éléments du paysage agraire de la région. Une analyse plus poussée, au niveau des paroisses, devrait nous faire découvrir le processus d'intégration de l'agriculture au marché.

Le choix des comtés exige sans doute d'être justifié. Il s'agit d'abord des deux comtés situés de part et d'autre de la rivière Saint-Maurice, lesquels forment ce que l'on peut appeler la Mauricie des débuts. Ce sont Champlain et Saint-Maurice (ce dernier pour les besoins de l'étude englobant le comté de Trois-Rivières qui connaît pendant une certaine période une existence autonome). S'y ajoute un troisième, celui de Nicolet - sur la rive opposée du Saint-Laurent, juste en face de la ville de TroisRivières - en raison des liens historiques que cette circonscription a entretenus avec la zone de Trois-Rivières. Domaine d'implantation ancienne, le comté de Nicolet offre une base de comparaison intéressante pour les deux autres comtés de la rive nord où la colonisation connaît un rythme nettement plus intense au 19e siècle. La Mauricie se définit bien par son épine dorsale, la rivière SaintMaurice. Ses contours sont cependant fluides. Nous nous sommes 
cru justifié de nous en tenir au périmètre de la Mauricie étroite pour y étudier les transformations de l'agriculture ${ }^{3}$.

Les données publiées des recensements ne peuvent que traduire imparfaitement l'évolution de l'agriculture au Québec, tellement les définitions, la terminologie et le mode même de l'enregistrement des données ont été modifiés de recensement en recensement ${ }^{4}$. Ces données doivent donc être interprétées avec beaucoup de précaution sous peine de déformations plus ou moins graves.

Par exemple, une partie des données des recensements de 1852,1861 et 1891 se rapportant aux superficies et aux volumes de production doivent être modifiées pour tenir compte d'une grave omission lors de leur publication. Dans les zones francophones, en général, les superficies ont été recensées en arpents et les volumes en minots. Or les clonnées publiées sont présentées en acres et en boisseaux, mais sarıs avoir été modifiées au préalable. Nous avons donc effectué cette conversion, en partie du moins. Nous avons aussi noté au passage certaines causes de distorsion particulièrement importantes. Malgré toutes les difficultés qu'elles posent, on peut penser que ces données demeurent indicatives des mouvements à long terme de l'agriculture. Pour notre part, en exprimant en pourcentage les données régionales par rapport à celles du Québec et les données relatives aux trois comtés par rapport à celles de la région, nous croyons échapper à l'effet de ces distorsions statistiques, les poids relatifs étant grossièrement les mêmes dans un système de comparaison où toutes les données souffrent des mêmes lacunes.

3 Le présent article se fonde en bonne partie sur L'agriculture en Mauricie... D'importantes corrections y ont été apportées cependant. 1) Toutes les données se rapportant aux sept paroisses qui en 1861 passent du comté de Saint-Maurice au comté de Maskinongé ont été éliminées pour l'année 1851 et les calculs refaits. Cette opération stabilise en gros la base territoriale de l'étude. Certes les trois comtés ont subi de nombreuses mutations territoriales durant cette longue période, mais il s'agit de mutations de faible portée à l'intérieur des cadres paroissiaux. Nous considérons qu'elles n'ont eu qu'un effet marginal de distorsion dans nos calculs. Sur les mutations territoriales, voir Jacques Henripin, «Les divisions de recensement au Canada de 1871 à 1951", (Actualité économique, no 4 (1955): 633-659; no 1 (1956): 102-127).

4 Des études importantes ont été publiées récemment sur cette délicate question pour la première partie du 19e siècle. Voir en particulier: F. Lewis et R.M. McInnis, «The Efficiency of the French-Canadian Farmer in the Nineteenth Century», The Journal of Economic History, XL, 3 (1980): 497-514; R.M. McInnis, "Some Pitfalls in the 1851-52 Census of Agriculture of Lower Canada», Histoire Sociale - Social History, XIV, 27 (1981): 219-231; R.M. Mclnnis, «A Reconsideration of the State of Agriculture in Lower Canada in the First Half of the Nineteenth Century", dans O.H. Akenson, Canadian Papers in Rural History, III (1982): 9-49; J. McCallum, Agriculture and Economic Development in Quebec and Ontario until 1870 (Toronto, UTP, 1980), 148 p.; Serge Courville, "La crise agricole du Bas-C'anada, éléments d'une réflexion géographique», Cahiers de géographie du Québec, 24, 62. (1980): 193-224 et 24, 63 (1980): 385-428; Allan Greer, «Fur Trade Labour and Lower Canadian Agrarian Structure», communication présentée au congrès de la Société historique du Canada de juin 1981, 33 p. 
On prendra cette analyse au ras du sol pour ce qu'elle est. Elle laisse à des travaux ultérieurs l'explication, par exemple, du processus d'intégration de l'agriculture au marché. Pour éviter d'alourdir le texte, nous avons renvoyé en annexe la présentation de la plupart des données sur lesquelles se fonde cet article.

\section{1 - LES GRANDS PARAMÈTRES DE L'AGRICULTURE QUÉBÉCOISE}

\section{- Les occupants}

Combien d'exploitants le Québec comptait-il entre 1852 et 1951? La question n'est pas simple. Pour y répondre il faudrait d'abord que nous puissions définir très exactement ce qu'était un exploitant. Malheureusement, nous ne disposons pas des informations nécessaires pour établir à toutes les périodes un partage entre les pseudo-exploitants (ceux qui possèdent la terre sans l'exploiter à des fins agricoles ou qui n'entretiennent qu'un potager), les semiexploitants qui pratiquent l'agriculture en général sur de petites surfaces comme une deuxième activité, comme un travail d'appui destiné à soutenir le revenu familial, et enfin les exploitants qui vivent principalement du produit de l'agriculture sur des terres généralement de moyenne et de grande surfaces.

La notion d'exploitation agricole fut précisée par ajustements successifs durant l'ensemble de la période étudiée ${ }^{5}$, mais nous avons cependant tenté de répartir les occupants en trois grandes catégories qui permettent de cerner l'évolution du nombre réel des exploitants: les occupants de 10 acres et moins, les occupants de 11 à 50 acres, et enfin les occupants de 50 acres et plus. Pour 1951, nous avons dû adopter un regroupement légèrement différent: les occupants de 9 acres et moins, ceux de 10 à 69 acres, et ceux de plus de 69 acres. Le tableau I présente ces trois catégories d'occupants pour l'ensemble du Québec et la Mauricie. Il paraît raisonnable de supposer que la masse des pseudo-exploitants qui encombrent plusieurs recensements agricoles, appartient à la première catégorie $^{6}$. Il paraît aussi raisonnable de supposer que la masse des semi-exploitants se retrouve dans les deux premières catégories. Quant à la troisième catégorie, on peut estimer qu'elle regroupe l'essentiel des exploitants vivant principalement de l'agriculture.

\footnotetext{
Voir à ce propos N. Séguin, R. Hardy et L. Verreault-Roy, L'agriculture en Mauricie..., 21-35.

C'est ce qu'a démontré R.M. McInnis pour le recensement de 1851-52 (voir son article intitulé «Some Pitfalls...», 221-222). Le recensement de 1901 fait état de 10489 lopins de moins d'un acre, celui de 1911, de 9990 (recensement de 1951, vol. 6, tableaux chronologiques, 1,2).
} 
Examinons le tableau I. On y constate d'abord que le nombre total des occupants de terres est jusqu'en 1911 fortement influencé par la proportion des occupants de la première catégorie. En effet, le haut sommet de 1891 et à un moindre niveau ceux de 1901 et de 1911 s'expliquent essentiellement par le gonflement de cette catégorie dont le déclin est très net à compter du recensement de 1921 . Si on fait abstraction de cette catégorie la croissance du nombre des exploitants est nettement plus modérée. En effet, la deuxième catégorie offre de 1851 à 1911 une remarquable stabilité qui précède un mouvement de régression. Quant à la troisième catégorie, la plus importante, celle qui englobe la majorité des exploitants vivant principalement de la terre, elle est en hausse constante jusqu'en 1941, année qui marque un sommet. À première vue, le recensement de 1951 révèle un recul considérable du nombre d'exploitants: plus de 20000 à l'échelle québécoise par rapport à 1941 . La réalité est cependant différente. En tenant compte du changement de définition, la baisse s'établit plutôt à 10000 environ?

\section{TABLEAU I \\ Nombre et répartition des occupants selon l'espace occupé au Québec, 1852-1951}

\begin{tabular}{|c|c|c|c|c|}
\hline Année & $\begin{array}{l}\text { Moins de } \\
10 \text { acres }\end{array}$ & $\begin{array}{l}\text { De } 11 \text { à } \\
50 \text { acres }\end{array}$ & $\begin{array}{l}\text { Plus de } \\
50 \text { acres }\end{array}$ & $\begin{array}{c}\text { Nombre } \\
\text { total }\end{array}$ \\
\hline 1852 & $14447(15 \%)$ & $20224(21.1 \%)$ & $61112(63.7 \%)$ & 95813 \\
\hline 1861 & $6822(6.4 \%)$ & $23260(22 \%)$ & $75589(71.5 \%)$ & 105671 \\
\hline 1871 & $10510(8.9 \%)$ & $22379(18.9 \%)$ & $85197(72.1 \%)$ & 118086 \\
\hline 1881 & $19150(13.8 \%)$ & $24564(17.8 \%)$ & $94149(68.2 \%)$ & 137863 \\
\hline 1891 & $51.057(29.1 \%)$ & $22296(12.7 \%)$ & $101643(58.0 \%)$ & 174996 \\
\hline 1901 & $24149(16.03 \%)$ & $20047(13.3 \%)$ & $106403(70.6 \%)$ & 150599 \\
\hline 1911 & $25962(16.25 \%)$ & $22209(13.9 \%)$ & $111520(69.8 \%)$ & 159691 \\
\hline 1921 & $7953(5.7 \%)$ & $17012(12.3 \%)$ & $112654(81.8 \%)$ & 137619 \\
\hline 1931 & $6710(4.9 \%)$ & $16976(12.4 \%)$ & $112271(82.5 \%)$ & 135947 \\
\hline \multirow[t]{2}{*}{1941} & $4413(2.8 \%)$ & $18875(12.2 \%)$ & $131381(84.9 \%)$ & 154669 \\
\hline & $\begin{array}{l}9 \text { acres } \\
\text { et moiris }\end{array}$ & $\begin{array}{l}\text { De } 10 \text { à } \\
69 \text { acres }\end{array}$ & $\begin{array}{l}\text { Plus de } \\
69 \text { acres }\end{array}$ & \\
\hline 1951 & $3004(2.2 \%)$ & $13289(9.8 \%)$ & $118043(87.8 \%)$ & 13433 \\
\hline
\end{tabular}

En effet, en prenant comme référence la définition de l'exploitation de 1951, le nombre des occupants au Québec en 1941 peut être ramené de 154669 à environ 145000 , ce qui coupe de moitié la baisse enregistrée durant la décennie: d'un peu plus de 20000 à environ 10000 , soit une diminution de près de 7\%. C'est l'estimation qu'ont avancée les responsables du recensement de 1951 (vol. 10, 456). 
Ce recul est quand même significatif. Le recensement de 1951 «annonce» l'érosion des effectifs de la paysannerie québécoise et confirme la marginalisation accrue des occupants de petites surfaces. Il est manifeste que le mode de dénombrement des exploitations en vigueur avant 1951 gonflait artificiellement le nombre d'exploitants dans une proportion indéterminée. On doit toujours en tenir compte.

Sur la base de la deuxième et de la troisième catégories, on peut affirmer que le nombre des exploitants vivant principalement de l'agriculture au Québec fut en hausse à peu près constante jusqu'en 1941. Leur nombre s'établit entre 61112 et 80000 en 1851 et entre 131381 et 150000 en 1941. Si par hypothèse on retient la moitié des occupants de 11 à 50 acres, on voit que le Québec aurait pu compter environ 70000 exploitants vivant principalement de la terre en 1851, et peut-être environ 140000 en 1941, soit une multiplication par deux en 90 ans.

\section{- L'espace agraire et les grandes productions végétales}

$\mathrm{Du}$ milieu du 19e siècle jusqu'au début du 20e, l'espace agraire québécois est en expansion. De façon constante, l'espace total occupé représente près du double de l'espace amélioré. Mais l'espace occupé n'est pas le meilleur indicateur des pulsations de l'oekoumène puisqu'il ne révèle pas directement l'ampleur des activités agricoles proprement dites. C'est l'espace amélioré qui rend compte le mieux de l'espace agraire utile, c'est-à-dire l'espace où s'exerce le labeur des exploitants dans l'agriculture. À l'échelle québécoise, l'espace amélioré enregistre des progrès soutenus jusqu'au recensement de 1921 et ne connaît par la suite que de faibles inflexions. Il en est ainsi de la superficie en grandes cultures, qui rythment en définitive les pulsations de l'oekoumène ${ }^{8}$. Les pâturages sont en hausse durant la seconde moitié du 19e siècle; ils régressent durant la première moitié du $20 \mathrm{e}^{9}$.

Ainsi, il y a stabilisation de l'espace agraire utile très tôt au $20 \mathrm{e}$ siècle et cela correspond au tassement des grandes productions végétales. Par leurs volumes de production et par la superficie qui leur est dévolue, le foin et l'avoine sont, et de très loin, les deux

8 Pour les fins de cette étude comparative, seul l'espace voué directement à l'agriculture est considéré. Certes le boisé est une composante importante, même essentielle, dans maintes exploitations. Mais il n'entrait pas dans notre propos de scruter les activités annexes qui s'y déroulent. Nous reviendrons dans une autre étude sur cette importante question.

$9 \quad$ Le mode d'enregistrement de la superficie en pâturage a beaucoup évolué d'un recensement à l'autre (voir N. Séguin, R. Hardy et L. Verreault-Roy, L'agriculture en Mauricie... 21-35). 
grandes productions végétales de l'agriculture québécoise contemporaine. Or pour toutes deux l'expansion cesse à l'aube du 20e siècle. En progrès rapide depuis le milieu du $19 \mathrm{e}$ siècle, la production de foin se stabilise après 1910. La production de l'avoine, elle, double entre 1851 et 1860 , et double presque à nouveau entre 1880 et 1900. Par la suite elle se maintient sensiblement au niveau atteint en 1900.

Le blé, une culture encore relativement importante au Québec en 1851 , est en déclin à peu près continu. Sa chute après $1920^{10}$ est particulièrement forte. Après cette date, elle devient vraiment une culture marginale. Le cas de l'orge est différent. Sa progression est très rapide entre 1851 et 1860 , puis c'est la relative stabilité jusqu'en 1940. Les données de 1950 indiquent pour cette culture un recul accusé au cours de la dernière décennie. Entre 1851 et 1870 , la production de la pomme de terre connaît également une hausse fort importante. Mais par la suite, à l'exception de l'année 1940 qui indique son plus haut sommet, son volume reste toujours inférieur à celui de 1870. Enfin, fait intéressant, le sarrasin, culture peu exigeante, enregistre de notoires progrès jusqu'au début du 20 e siècle, avant d'amorcer un déclin graduel assez prononcé jusqu'en 1950.

Pour cette période, l'évolution différentielle des grandes productions végétales marque profondément l'espace agraire. En 1851 , l'avoine accapare $28,5 \%$ de l'espace en culture, et $23,5 \%$ en 1950; le foin (la superficie qui lui est réservée peut être évaluée entre 600000 et 800000 acres en 1851 et en 1860) retient entre 30 et $40 \%$ de l'espace cultivé en 1851 , et près de $60 \%$ en 1950 . Ainsi, plus que toute autre grande production végétale, le foin détermine l'évolution de l'espace cultivé dans l'agriculture contemporaine du Québec.

\section{- Les grandes productions animales}

La forte croissance du nombre de vaches laitières, de porcs et de volailles, est le fait majeur de l'histoire des grandes productions animales entre 1852 et 1951.

10 Dans leur Histoire économique du Québec, 1851-1896, (Montréal, Fides, 1971, 200) J. Hamelin et Y. Roby, estimant que le blé ne représente plus que 3.0\% des emblavures en concluent à sa disparition. Comme le montrent les données de l'annexe III, c'est après 1920 seulement que l'on peut parler de la disparition relative du blé dans le paysage agraire québécois. La production du blé en 1900 équivaut encore aux deux tiers de celle de 1851 . 
L'élevage laitier ne cesse de croître; il double durant la seconde moitié du $19 \mathrm{e}$ siècle et double de nouveau durant les quatre premières décennies du 20e. Entre 1941 et 1951, il accuse toutefois une diminution de 100000 têtes (moins de 10\%). L'élevage porcin, qui en 1852 n'est que légèrement inférieur en nombre de têtes à l'élevage laitier, affiche une croissance beaucoup plus lente que celui-ci jusqu'au tournant du siècle. Puis, c'est la hausse fulgurante. Le nombre de têtes est presque multiplié par deux entre 1901 et 1911. Ce plateau est maintenu en gros jusqu'au recensement de 1941. En 1951, avec 300000 têtes de plus, le troupeau de porcs dépasse même légèrement celui des vaches laitières. Quant aux volailles, depuis 1891, année des premières données disponibles, leur nombre s'accroît vigoureusement par paliers. La progression est de plus de 2000000 de têtes entre 1941 et 1951. En soixante ans l'élevage est passé de près de trois millions de têtes à plus de dix millions.

La décroissance des ovins et des chevaux contraste avec ces fortes poussées au 20e siècle. En ce qui a trait à l'élevage ovin, le plus important numériquement au milieu du 19e siècle, son évolution est bien particulière. Sauf en 1871, l'année du plus haut sommet, et en 1881 et en 1921 , le troupeau ovin ne s'éloigne guère du niveau de 1851 jusqu'au recensement de 1931. Il subit par contre en 1941 et en 1951 une baisse abrupte. Entre le recensement de 1931 et celui de 1951, la réduction du nombre de têtes est supérieure à $50 \%$. Enfin, le nombre de chevaux au Québec double presque entre 1851 et 1891 , puis il varie modérément jusqu'en 1941 . Mais entre 1941 et 1951, il diminue du tiers. L'ère des chevaux est d'ores et déjà révolue.

\section{2 - LA PLACE DE LA MAURICIE DANS L'AGRICULTURE QUÉBÉCOISE}

\section{- Les occupants}

La répartition des occupants en trois catégories montre un profil régional qui s'écarte très peu du profil québécois. 


\begin{tabular}{|c|c|c|c|c|c|}
\hline \multirow[b]{2}{*}{ Année } & \multicolumn{5}{|c|}{$\begin{array}{c}\text { TABLEAU II } \\
\text { Nombre et répartition des occupants } \\
\text { selon l'espace occupé en Mauricie, 1852-1951 }\end{array}$} \\
\hline & $\begin{array}{l}\text { Moins de } \\
10 \text { acres }\end{array}$ & $\begin{array}{l}\text { De } 11 \text { à } \\
50 \text { acres }\end{array}$ & $\begin{array}{l}\text { Plus de } \\
50 \text { acres }\end{array}$ & $\begin{array}{c}\text { Nombre } \\
\text { total }\end{array}$ & $\begin{array}{l}\mathbf{M} / \mathbf{Q} \\
\text { en } \%\end{array}$ \\
\hline 1852 & $733(12.8 \%)$ & $1002(17.6 \%)$ & $3748(65.9 \%)$ & 5683 & 5.9 \\
\hline 1861 & $113(1.8 \%)$ & $1617(26 \%)$ & $4471(72.1 \%)$ & 6198 & 5.9 \\
\hline 1871 & $490(6.6 \%)$ & $1399(19.1 \%)$ & $5424(74.0 \%)$ & 7322 & 6.2 \\
\hline 1881 & $884(10.3 \%)$ & $1645(19.2 \%)$ & $6037(70.4 \%)$ & 8566 & 6.2 \\
\hline 1891 & $3226(28.1 \%)$ & $1359(11.8 \%)$ & $6878(60.0 \%)$ & 11463 & 6.6 \\
\hline 1901 & $1904(18.7 \%)$ & $997(9.8 \%)$ & $7263(71.4 \%)$ & 10164 & 6.7 \\
\hline 1911 & $1957(17.8 \%)$ & $1195(10.9 \%)$ & $7789(71.19 \%)$ & 10941 & 6.8 \\
\hline 1921 & $409(4.8 \%)$ & $817(9.6 \%)$ & $7224(85.3 \%)$ & 8462 & 6.1 \\
\hline 1931 & - & - & - & 7998 & 5.9 \\
\hline \multirow[t]{2}{*}{1941} & $187(2.2 \%)$ & $890(10.5 \%)$ & $7341(87.2 \%)$ & 8418 & 5.4 \\
\hline & $\begin{array}{l}9 \text { acres } \\
\text { et moins }\end{array}$ & $\begin{array}{l}\text { De } 10 \text { à } \\
69 \text { acres }\end{array}$ & $\begin{array}{l}\text { Plus de } \\
69 \text { acres }\end{array}$ & & \\
\hline 1951 & $84(1.1 \%)$ & $1123(15.5 \%)$ & $6017(83.2 \%)$ & 7224 & 5.4 \\
\hline
\end{tabular}

Comme pour l'ensemble québécois, l'élément majeur dans l'évolution du nombre des occupants régionaux, c'est l'accroissement constant des occupants de 50 acres et plus: plus de $65 \%$ en 1852 , plus de $83 \%$ en 1951 (occupants de 69 acres et plus). En retranchant du nombre total des occupants de la région ceux de la catégorie des 10 acres et moins, nous constatons que le nombre d'exploitants vivant principalement de la terre s'établit entre 3748 et 4750 en 1852 et entre 6017 et 7140 en 1951 . Peut-être ne serionsnous pas éloignés de la réalité en disant que la Mauricie comptait un peu plus de 4000 occupants de ces deux catégories en 1852, et un peu moins de 7000 en 1951. Au sommet de 1911, la région a pu en compter environ 8000 .

Toutes catégories confondues, on constate que le poids des occupants de la Mauricie dans l'ensemble québécois demeure passablement stable durant tout le siècle: $5,9 \%$ en $1852,5,4 \%$ en 1951. En fait, la colonisation et l'extention de l'agriculture marginale favorisent au $19 \mathrm{e}$ siècle la multiplication rapide des occupants dans la région, d'où le sommet de $6,8 \%$ en 1911 . Au vingtième siècle toutefois, le mouvement d'intégration de l'agriculture au marché a l'effet inverse, d'où le déclin relatif du poids des occupants régionaux jusqu'en 1951. 


\section{- L'espace agraire et les grandes productions végétales}

Exprimé en pourcentage du total québécois, l'espace occupé en Mauricie a tendance à décroître au 20e siècle, et davantage en fin de période. Mais il ne faut pas en conclure que la position de l'agriculture régionale dans l'ensemble québécois se détériore. Au contraire, les autres indicateurs que sont l'espace amélioré, l'espace en grandes cultures et les pâturages montrent un poids régional plus considérable durant la première moitié du 20e siècle que durant la seconde moitié du 19e siècle. Une des faiblesses de l'agriculture régionale au $19 \mathrm{e}$ siècle, et cela influe sur l'espace amélioré, c'est la dimension réduite des pâturages. Le $20 \mathrm{e}$ siècle corrigera cette déficience. On le constate donc, durant la période de colonisation, l'espace agraire régional évolue moins rapidement que la masse des occupants. On assiste donc à un important redressement des principales caractéristiques de l'espace agraire régional, et surtout des pâturages. Celui-ci est d'ailleurs concomitant de la diminution du nombre total d'occupants. Le profil des grandes caractéristiques de l'espace agraire établi sur la base de l'occupant moyen rend bien compte de ce redressement, ainsi qu'on peut le voir dans le tableau III.

\section{TABLEAU III \\ Évolution de l'utilisation du sol (acres) Moyenne par occupant au Québec et en Mauricie}

\begin{tabular}{|c|c|c|c|c|c|c|c|c|}
\hline \multirow[t]{2}{*}{ Année } & \multicolumn{2}{|c|}{ Espace occupé } & \multicolumn{2}{|c|}{ Espace amélioré } & \multicolumn{2}{|c|}{ Esp. en gr. cult. } & \multicolumn{2}{|c|}{ Esp. en pâturage } \\
\hline & $\mathrm{Q}$ & M & $\mathrm{Q}$ & M & $\mathrm{Q}$ & $\mathrm{M}$ & $\mathrm{Q}$ & $\mathrm{M}$ \\
\hline 1852 & 74.8 & 75.0 & 32.8 & 26.3 & 19.3 & 16.3 & 13.7 & 9.8 \\
\hline 1861 & 87.6 & 80.9 & 39.9 & 31.3 & 24.3 & 21.0 & 15.3 & 10.1 \\
\hline 1871 & 93.4 & 89.6 & 48.3 & 38.7 & 31.5 & 26.1 & 16.5 & 12.3 \\
\hline 1881 & 91.5 & 90.5 & 46.5 & 39.9 & 30.1 & 27.2 & 16.1 & 12.4 \\
\hline 1891 & - & 70.0 & - & 35.6 & - & 24.5 & - & 10.7 \\
\hline 1901 & 95.9 & 82.8 & 49.4 & 42.7 & 31.2 & 27.5 & 22.3 & 15.9 \\
\hline 1911 & 97.7 & 83.0 & 51.1 & 47.3 & 34.3 & 31.3 & - & - \\
\hline 1921 & 125.4 & 114.1 & 65.9 & 67.9 & 43.3 & 45.6 & 22.3 & 21.0 \\
\hline 1931 & 127.3 & 113.5 & 66.2 & 69.7 & 44.7 & 47.1 & 21.0 & 20.4 \\
\hline 1941 & 116.8 & 104.9 & 58.6 & 65.5 & 39.2 & 43.9 & 18.9 & 18.9 \\
\hline 1951 & 125.0 & 115.1 & 65.7 & 70.0 & 42.3 & 46.5 & 22.6 & 20.7 \\
\hline
\end{tabular}

S'agissant des grandes cultures, la Mauricie se caractérise au $19 \mathrm{e}$ siècle par sa participation très élevée à la production québécoise de l'avoine et du sarrasin (entre $7,3 \%$ et $8,2 \%$ pour le premier et entre $6,9 \%$ et $10,8 \%$ pour le second). Les autres grandes 
productions conservent pour la plupart un poids régional inférieur à celui de la masse des occupants: en ordre décroissant, on retrouve le foin, le blé, la pomme de terre et enfin l'orge. Sans doute, l'importance considérable accordée à la production de l'avoine et du sarrasin durant ce demi-siècle est-elle à mettre en rapport avec l'exploitation forestière et l'expansion du front pionnier vers les marges de l'oekoumène, où le sarrasin s'accommode de sols de faible potentialité.

Ce profil est passablement modifié au cours de la première moitié du 20e siècle. La Mauricie diminue sa participation dans la production québécoise de l'avoine, en lui conservant toutefois un poids relativement élevé $(6,2 \%$ en 1950). Et elle délaisse plus rapidement que l'ensemble du Québec le blé et le sarrasin (respectivement $1,1 \%$ et $3,8 \%$ de la production québécoise en 1950). Par contre, elle accroît sa participation dans la production de l'orge (dont le poids demeure néanmoins encore modeste, 3,0\% en 1950) et de la pomme de terre, qui tend à s'imposer comme une des grandes cultures de la Mauricie (7,4\% en 1950). Sauf en 1920, où elle atteint $7 \%$ de la production québécoise, la culture du foin dans la région ne ressort ni particulièrement forte ni particulièrement faible. Ce nouvel équilibre dans les grandes productions végétales trouve son pendant dans les productions animales.

\section{- Les grandes productions animales}

La composition des cheptels de la Mauricie durant la seconde moitié du 19e siècle est une autre indication de la faiblesse de l'agriculture régionale. Le troupeau des ovins est alors le troupeau régional dont le poids est le plus lourd dans l'ensemble québécois; il équivaut à peu près à celui de la masse des exploitants. Vient ensuite le troupeau de porcs. Comme pour l'avoine et le sarrasin, l'importance qu'accorde la région à l'élevage des ovins et des porcs tient sans doute en partie à l'exploitation forestière et l'extension de l'agriculture pionnière. Le troupeau de vaches laitières est plutôt modeste et n'enregistre que de légers progrès vers la fin du siècle $(5,7 \%$ du total québécois en 1901). Quant aux chevaux, leur proportion est la plus faible: toujours inférieure à 5,0\%. L'agriculture mauricienne de la seconde moitié du 19e siècle se caractérise par la relative pauvreté de ses cheptels.

Il en va autrement durant le demi-siècle qui suit. La Mauricie délaisse l'élevage ovin, plus rapidement qu'ailleurs au Québec. Parallèlement elle accroît sa part dans les autres élevages: l'élevage laitier, bien sûr, mais davantage l'évelage porcin. La Mauricie obtient aussi une proportion accrue de chevaux dans l'ensemble québécois. Sans doute faut-il y voir la conséquence du retard de la motorisation de l'agriculture régionale avant la charnière de 1950. 
Comme ailleurs au Québec, la montée rapide de l'élevage des volailles devient une des grandes caractéristiques de l'agriculture régionale au 20e siècle.

Vers 1951, compte tenu du nombre d'exploitants, la Mauricie dispose de cheptels imposants. Alors que les exploitants mauriciens ne représentent plus que $5,4 \%$ des exploitants québécois, ils disposent de 5,8\% des vaches laitières, de 6,5\% des porcs et de $5,6 \%$ des volailles. La région fait bonne figure dans les grandes productions animales de marché. C'est ce que l'on peut constater dans le tableau IV.

\section{TABLEAU IV \\ Évolution des grandes productions animales au Québec et en Mauricie}

Nombre moyen de têtes par occupant

\begin{tabular}{lrrrrrr} 
Année & \multicolumn{2}{c}{ Vaches laitières } & \multicolumn{2}{c}{ Moutons } & \multicolumn{2}{c}{ Porcs } \\
& $\mathrm{Q}$ & $\mathrm{M}$ & $\mathrm{Q}$ & $\mathrm{M}$ & $\mathrm{Q}$ & $\mathrm{M}$ \\
1852 & 3.1 & 3.2 & 6.8 & 6.9 & 2.7 & 2.7 \\
1861 & 3.1 & 2.8 & 6.5 & 6.2 & 2.7 & 2.5 \\
1871 & 3.4 & 3.4 & 8.5 & 10.0 & 3.1 & 3.4 \\
1881 & 3.5 & 2.9 & 6.4 & 6.6 & 2.3 & 2.2 \\
1891 & 3.1 & 2.5 & 4.2 & 4.1 & 2.1 & 1.9 \\
1901 & 5.0 & 4.2 & 4.3 & 4.2 & 2.7 & 2.5 \\
1911 & 4.7 & 4.3 & 4.3 & 3.4 & 5.0 & 4.7
\end{tabular}

Nombre moyen de têtes par occupant déclarant faire le dit élevage

\begin{tabular}{rrrrrrr}
1921 & 6.6 & 6.4 & 12.8 & 11.1 & 6.6 & 6.8 \\
1931 & 7.7 & 7.6 & 14.2 & 13.5 & 7.5 & 8.0 \\
1941 & 9.4 & 9.4 & 10.6 & 9.4 & 7.5 & 7.6 \\
1951 & - & 10.3 & 11.4 & 9.8 & 12.5 & 14.4 \\
\hline
\end{tabular}

\section{3) L'ÉVOLUTION DE L'AGRICULTURE DANS LES TROIS COMTÉS DE LA MAURICIE}

Pour ce qui est de capacité de production agricole, Nicolet est le comté le mieux nanti de la région et fait même bonne figure dans l'ensemble québécois. Quant aux comtés de la rive nord, Champlain et Saint-Maurice, la géographie s'y fait plus capricieuse et y impose de nombreuses restrictions aux activités agricoles. À ce titre, le comté de Saint-Maurice sur la rive droite du Saint-Maurice 
est le moins bien pourvu de la région. Cela étant, la répartition spatiale des activités agricoles à l'intérieur de la Mauricie laisse voir des cheminements très contrastés de l'agriculture dans les trois comtés de la région tout au long de la période étudiée. Le mouvement de colonisation et le processus d'intégration de l'agriculture au marché les marquent différemment.

\section{- Les occupants}

Entre 1852 et 1951, le comté de Nicolet représente toujours le plus grand nombre d'occupants de la région. Vers 1850 il est fort de plus de 45\% des occupants de la Mauricie. En 1911, il en retient un peu moins de 39\%. C'est que le mouvement de colonisation a profité davantage aux comtés de la rive nord, et surtout au comté de Champlain dont les occupants sont passés de près de $33 \%$ du total régional en 1852 à un peu plus de $38 \%$ en 1911 . Le comté de Saint-Maurice est clemeuré, lui, à peu près stable autour de $22 \%$. Durant la première moitié du 20e siècle, le comté de Nicolet obtient une proportion accrue des occupants de la région: un peu plus de $44 \%$ en 1951; il retrouve alors sa position de 1852 . Entretemps, les deux comtés de la rive nord ont reculé: Champlain à $36,7 \%$ et Saint-Maurice à $19,0 \%$. Ces mouvements d'expansion et de contraction du nombre d'occupants de part et d'autre du SaintLaurent soulignent éloquemment les grandes tendances du domaine agricole régionale aux $19 \mathrm{e}$ et $20 \mathrm{e}$ siècles.

Si l'on répartit les occupants de la Mauricie selon les trois grandes catégories que nous avons définies au début de cet article, on peut alors constater la relative vigueur du comté de Nicolet au $19 \mathrm{e}$ siècle aussi bien qu'au 20e, et la non moins évidente fragilité du comté de Saint-Maurice. En effet, non seulement le comté de Nicolet conserve-t-il toujours la portion la plus considérable des occupants de la région, mais plus encore, il accapare d'une décennie à l'autre le nombre le plus grand des occupants de la troisième catégorie, ceux de 50 acres et plus. À l'opposé, le comté de SaintMaurice obtient ses proportions les plus élevées d'occupants régionaux dans les première et deuxième catégories, c'est-à-dire, celles des occupants de 10 acres et moins et de 10 à 50 (69) acres. Quant au comté de Champlain, ses plus fortes proportions sont obtenues dans la deuxième catégorie. En somme, en dépit des fluctuations dans la répartition régionale du nombre des occupants, Nicolet demeure toujours le grand comté agricole de la Mauricie, et il se retrouve plus fort au $20 \mathrm{e}$ siècle qu'au $19 \mathrm{e}$ siècle. L'analyse des grandes caractéristiques de l'espace agraire, des grandes productions végétales et animales le démontre. 


\section{- L'espace agraire et les grandes productions végétales}

C'est sous la rubrique de l'espace occupé que l'on mesure le mieux l'impact de la forte poussée de la colonisation sur la rive nord. Durant la seconde moitié du 19e siècle, le comté de Champlain retient une part plus grande de l'espace régional occupé que le comté de Nicolet. Au 20e siècle, la situation est inversée. En 1951, la part de ce dernier dans l'espace régional occupé correspond néanmoins tout juste à la masse de ses occupants. L'espace amélioré singularise davantage l'évolution du comté de Nicolet et il nous donne une plus juste mesure de son poids réel dans l'agriculture régionale. Que ce soit au chapitre de l'espace en grandes cultures, des pâturages ou de l'espace total amélioré, la part la plus substantielle revient toujours au comté de Nicolet à près de $40 \%$ au moins, et elle s'accroît notablement frôlant les 50\% vers 1950 . Sur la rive nord, le comté de Champlain enregistre d'importants progrès jusqu'à la fin du $19 \mathrm{e}$ siècle (sa part de l'espace amélioré dans la région passe de $30 \%$ environ en 1852 à plus de $38 \%$ en 1901), mais il y a contraction par la suite. En 1951, il arrive très loin derrière le comté de la rive sud avec à peine le tiers de l'espace régional amélioré. Quant au comté de Saint-Maurice, entre 1850 et 1870 environ, il amorce un mouvement de contraction continu qui tend à le marginaliser dans l'espace régional: $27,4 \%$ de l'espace amélioré à son sommet de $1861,20,7 \%$ en 1901 et seulement $18,6 \%$ en 1951 , en fin de période. L'examen des grandes cultures aux $19 \mathrm{e}$ et $20 \mathrm{e}$ siècles nous permet de mieux saisir le profil particulier de chacun des comtés de la région. Dans un siècle comme dans l'autre, elles sont très inégalement réparties dans l'espace régional et opposent fortement la rive nord à la rive sud du Saint-Laurent. Le comté de Nicolet, dont la capacité de production agricole est la plus grande, dispose aussi, comme nous le verrons plus loin, des cheptels les plus imposants. Et c'est là que se concentre massivement la production du foin et du blé, à plus de $40 \%$ au moins. Et dans le cas du foin, cette proportion tend à augmenter; elle atteint près de $54 \%$ en 1950 . Nous négligeons ici le blé qui au vingtième siècle devient rapidement une production marginale. Il en va autrement pour les autres cultures.

Le sarrasin, culture relativement peu exigeante, est davantage cultivé sur la rive nord, et surtout dans le comté de Saint-Maurice, le moins bien pourvu de la région pour ce qui est de la potentialité agricole. C'est d'ailleurs cette culture qui donne au comté de SaintMaurice ses taux de participation les plus élevés aux grandes cultures régionales: entre le quart et le tiers de la production de sarrasin, ce qui est nettement supérieur au poids de ses occupants. Le comté de Nicolet délaisse plus rapidement cette culture déclinante que ses vis-à-vis de la rive nord: même pas $30 \%$ en 1950, contre plus de 
$44 \%$ pour le comté de Champlain et plus de $26 \%$ pour le comté de Saint-Maurice.

Voyons maintenant l'avoine. Jusque vers 1930, le comté de Champlain s'impose comme le grand producteur d'avoine, dépassant le comté de Nicolet avec plus de $40 \%$ de la production régionale. Nous croyons qu'il s'agit là d'une conséquence des relations étroites que ce comté entretenait avec l'exploitation forestière, dont les besoins d'avoine étaient considérables avant la motorisation. Vers 1950, le comté de Nicolet déclasse nettement le comté de Champlain dans cette production à laquelle il contribue à près de $50 \%$.

Le cas de la pomme de terre s'apparente à celui de l'avoine. En 1851, le comté de Nicolet possède un peu plus de la moitié de la superficie consacrée à cette culture dans la région. Graduellement cette proportion baisse à $35 \%$ en 1900 . Elle remonte et atteint à nouveau plus de la moitié en 1950 après certaines fluctuations. Dans le comté de Champlain, la part de la production régionale de pommes de terre s'élève à près de $45 \%$ en 1900 et décline ensuite à près de $30 \%$ en 1950. Dans le comté de Saint-Maurice la production de cette denrée s'établit aux environs de $20 \%$ pour l'ensemble de la période jusqu'en 1940, puis elle tombe brusquement à près de $10 \%$. La culture de la pomme de terre s'adapte donc à la demande croissante des centres urbains en pleine expansion, ce qui détermine la remontée du comté de Nicolet dans cette production régionale.

Avec la moitié de la production du foin, de l'avoine et des pommes de terre en 1950, le comté de Nicolet domine sans conteste les grandes cultures régionales de marché, laissant loin derrière lui les comtés de la rive nord.

\section{- Les grandes productions animales}

Tout comme les productions végétales, les productions animales soulignent la prééminence du comté de Nicolet dans l'ensemble régional. Il s'agit du comté laitier de la région: près de $50 \%$ des têtes recensées en 1852. Entre cette date et 1871, le troupeau laitier subit une baisse de près de $10 \%$ du total régional. Puis, reprenant un mouvement à la hausse, il retrouve en 1911 à peu près la proportion de 1852, et la dépasse sensiblement par la suite, atteignant $52,1 \%$ en 1951 . Le cas du porc est également révélateur. Jusqu'en 1911, le troupeau porcin du comté de Nicolet varie entre $30,9 \%$ et $39,5 \%$ du total régional; puis il fait un bond à $42,4 \%$ en 1921 , à $52,1 \%$ en 1941 et à $61,9 \%$ en 1951 . Tout aussi instructif est le cas du mouton qui occupe $50 \%$ du total régional en 1852 et qui 
par étapes est ramené à un maigre 18,9\% en 1951. L'élevage des volailles accroît lui aussi l'importance du comté de Nicolet à compter de 1921. On le voit très bien, le comté de Nicolet domine nettement dans les élevages qui aux $19 \mathrm{e}$ et au 20e siècles correspondent aux grandes orientations de l'agriculture québécoise: l'élevage laitier d'abord, rejoint bientôt par l'élevage porcin, et enfin par celui des volailles.

Sur la rive nord, la position du comté de Saint-Maurice ne cesse de reculer après 1871 dans le cas de l'élevage laitier. Il s'agit de l'élevage le moins affirmé du comté: même pas $16 \%$ du total régional en 1951. La situation semble un peu meilleure pour l'élevage porcin. Néanmoins la part du comté de Saint-Maurice dans cet élevage régional fait une chute considérable entre 1941 et 1951. Elle ne représente plus que $15 \%$ environ en fin de période. Dans ce comté, l'élevage du mouton attire nettement l'attention. En légère régression par rapport à l'ensemble régional à la fin du 19e siècle, il est en hausse au 20e siècle et conserve un niveau élevé. Bref, le nombre de moutons diminue moins rapidement dans ce comté que dans les deux autres. Plus de 43\% du cheptel ovin régional en 1951 lui appartient. L'élevage des volailles progresse en fin de période. Dans Champlain, le comté voisin, le poids relatif du troupeau porcin, du troupeau laitier et du troupeau ovin dans l'ensemble régional affiche une hausse à peu près continue jusqu'au début du $20 \mathrm{e}$ siècle alors qu'il régresse durant les décennies suivantes. Il en va de même pour l'élevage des volailles qui diminue continuellement entre 1891 et 1951. Un autre constat s'impose ici. Au 19e siècle, le comté de Champlain tend à dominer dans les élevages qui correspondent à l'économie des fronts pionniers (le cheptel ovin) et à l'économie forestière (le cheptel porcin). Au siècle suivant, il est nettement déclassé dans les élevages où se confirment les grandes orientations de l'agriculture québécoise.

Pour ce qui est des chevaux, les comtés de la rive nord obtiennent des proportions plus fortes du total régional au 19e siècle qu'au 20e: un sommet de près de $40 \%$ dans le comté de Champlain en 1901 et un sommet de 24,4\% dans le comté de Saint-Maurice en 1911. Durant la première moitié de ce siècle, la part du comté de Nicolet ne cesse de s'élever: près de $48 \%$ en 1951 contre $34,3 \%$ pour le comté de Champlain et $18,2 \%$ pour le comté de SaintMaurice. Sans doute faut-il y voir un lien entre l'exploitation forestière de la haute Mauricie et la part relativement importante des comtés de la rive nord dans le nombre de chevaux. En 1951, comme dans le cas des grandes productions végétales, le comté de Nicolet s'approprie au moins la moitié des grands élevages à l'exception de ceux du mouton et des volailles. 


\section{Conclusion}

Entre 1850 et 1950, l'agriculture québécoise a traversé deux grandes phases. Une première qui s'étend jusqu'au début du $20 \mathrm{e}$ siècle, dominée par l'expansion de grandes cultures, surtout celles $\mathrm{du}$ foin et de l'avoine. Une seconde qui remonte aux dernières décennies du $19 \mathrm{e}$ siècle et couvre toute la première moitié du $20 \mathrm{e}$ siècle, dominée, elle, par la croissance de grands élevages de marché: vaches laitières, porcs, volailles, essentiellement. La décennie de 1940 montre même une accélération qui prélude à la phase agroindustrielle actuelle instaurée au cours de la décennie suivante. Mais, par rapport aux formidables chambardements que connaîtra l'agriculture québécoise dans le système agro-industriel (érosion rapide du nombre d'exploitants, agrandissement spectaculaire des exploitations, élargissement sans précédent de la taille des élevages...), on ne peut se départir d'une certaine impression de lenteur à propos du processus de transformation de l'exploitation québécoise entre le milieu du siècle précédent et les années 1950. Ainsi, pendant que les exploitants se multiplient rapidement, l'exploitation québécoise se transforme peu, en dépit du processus d'ajustement des productions au marché. C'est en définitive lorsque l'agriculture cesse d'absorber l'afflux de nouvelles générations qu'elle amorce sa conversion en profondeur.

L'agriculture mauricienne emprunte la même voie, mais elle évolue à un rythme spécifique. Durant la seconde moitié du $19 \mathrm{e}$ siècle, propulsé par un mouvement intense de colonisation, le nombre d'exploitants y grandit plus rapidement que dans l'ensemble du Québec. Mais le mouvement de colonisation qui annexe d'importantes quantités de terres neuves à l'oekoumène, lieu par excellence d'une production lente à s'articuler au marché, déprime l'agriculture régionale dans l'ensemble québécois dont les progrès sont plus rapides. Au 20e siècle, c'est le cheminement inverse. Pendant que le poids des exploitants régionaux diminue sensiblernent, l'agriculture mauricienne s'affirme avec une certaine vigueur.

Ces deux phases du cycle de l'agriculture mauricienne marquent différemment les grands blocs de l'espace agraire régional. Certes, tout au long du siècle étudié, le comté de Nicolet en demeure le coeur. Néanmoins, durant la seconde moitié du 19e siècle, la colonisation tend à déplacer le centre de gravité des effectifs agricoles au profit de la rive nord, où l'agriculture des fronts pionniers et la proximité de l'exploitation forestière impriment aux productions des inflexions qui contrastent nettement avec la situation observée sur la rive sud. Au 20e siècle, on assiste à un net recul de la rive nord dans l'ensemble mauricien. S'intégrant mieux au mar- 
ché, le comté de Nicolet est le véritable moteur de la remontée de l'agriculture régionale dans l'ensemble du Québec.

Cette réflexion sur une partie des données agricoles du Québec et de la Mauricie, remet en question toute vision monolithique de l'agriculture québécoise et des agricultures dites régionales en particulier. Elle nous invite à reconsidérer les productions agricoles d'un point à un autre de l'espace québécois. Pour nous, elle constitue une étape dans l'étude de la question agraire, une étape nécessaire pour éclairer convenablement les bases matérielles sur lesquelles se fonde l'existence de la paysannerie mauricienne. Concrètement, il nous fallait, dans un premier temps, dégager les structures spatiales de l'agriculture mauricienne. Cette démarche a non seulement l'avantage de nous aider à mieux évaluer les transformations de l'agriculture en Mauricie, mais elle rend aussi possibles des comparaisons avec les autres régions du Québec. L'étude de trois paroisses témoins, que nous citions en introduction, constitue la prochaine étape dans la découverte des grandes transformations de l'agriculture régionale et surtout des mécanismes qui orientent son devenir. Elle permettra de faire ressortir encore davantage les nuances qui nous aideront à mieux saisir la diversité des situations dans l'espace régional.

\section{ANNEXES}

Note: La conversion des données de 1852 a été faite à partir de la grille de correction mise au point par R.M. McInnis (voir son article intitulé: «Some Pitfalls...»). Nous y avons apporté de nécessaires corrections. Pour le recensement de 1861 , la conversion a été faite essentiellement à partir de la grille McInnis telle que corrigée par nous. Pour les paroisses qui en 1861 n'apparaissent pas dans la grille de 1851-52, nous nous sommes guidé sur les corrections effectuées sous la responsabilité de J.-C. Taché lors de la publication des données du recensement de 1871 . Les responsables du recensement de 1901 ont corrigé en bonne partie les données déficientes du recensement de 1891. Nous disons en bonne partie parce que la correction a porté uniquement sur les cultures proprement dites. Les données se rapportant à la superficie occupée, à la superficie améliorée, à la superficie non améliorée et aux pâturages ont échappé à cet effort de transformation. Malheureusement, les corrections effectuées n'ont pas fait l'objet en 1901 d'une présentation détaillée, comté par comté. Seules ont été retenues pour publication les données relatives à l'ensemble québécois pour les différentes cultures (Recensement de 1901, vol. 2: XVIII). Ce sont ces données corrigées qui figurent dans nos tableaux. Nous avons ignoré les données non corrigées de 1891, ci-haut mentionnées et se rapportant à l'ensemble québécois, jugeant encore insuffisantes les bases pour en faire la conversion. Nous avons pu cependant mener ce travail au niveau régional (voir N. Séguin, R. Hardy et L. Verreault-Roy, L'agriculture en Mauricie..., 26-27). Dans les recensements antérieurs à celui de 1901, la superficie en culture a été établie simplement en soustrayant de la superficie améliorée déclarée la superficie en pâturages, en vergers et en jardins. Á compter de 1901 la superficie en culture correspond à la somme des superficies des différentes cultures recensées. Les responsables du recensement de 1901 se sont livrés à un travail de reconstitution de la superficie en culture en 1891 et ont constaté 
un écart extrêmement considérable entre le résultat de leur calcul (4 064716 acres pour l'ensemble québécois après conversion des arpents en acres) et l'estimation de 1891 de 5542780 acres. Ils en ont clonc déduit que la superficie en culture indiquée dans les autres recensements de 1871 et 1881 dépasse la réalité (Recensement de 1901, vol. 2, XIV). On peut en dire autant sans doute des recensements de 1852 et de 1861 . On notera toutefois que le récapitulatif de 1941 donne pour 1891 une superficie en culture de 4610587 acres. Ce nombre se rapproche sensiblement du résultat que nous obtiendrions en corrigeant simplement l'estimation de 1891 de 5542780 acres pour tenir compte des arpents qui doivent être changés en acres. Nous avons retenu la correction de 1901 qui nous paraît plus conforme à la réalité. Cela étant, le lecteur se gardera de conclure à une baisse de la superficie en culture entre 1881 et 1891 . Il est même fort probable qu'il y ait une hausse importante.

En indiquant l'année du recensement pour les données se rapportant aux grandes caractéristiques de l'espace agraire nous suivons le mode de présentation adopté dans le récapitulatif de 1951 .

Les données revisées de 1852 et 1861 que nous présentons dans les annexes I et II ne sont encore que des approximations. Nous les tenons comme provisoires en attendant de pouvoir tenter un traitement plus poussé. Dans une prochaine note de recherche préparée en collaboration avec Jean-Claude Robert et portant sur certaines difficultés que posent ces recensements agricoles, nous exposerons le traitement des données que nous avons effectué.

\begin{tabular}{|c|c|c|c|c|}
\hline \multicolumn{5}{|c|}{$\begin{array}{c}(A N N E X E I)-L \text { 'espace agraire, acres } \\
\text { QUÉBEC }\end{array}$} \\
\hline Année & $\begin{array}{l}\text { Superficie } \\
\text { occupée }\end{array}$ & $\begin{array}{l}\text { Superficie } \\
\text { améliorée }\end{array}$ & $\begin{array}{l}\text { Superficie en } \\
\text { gr. cultures }\end{array}$ & $\begin{array}{c}\text { Superficie } \\
\text { en pâturage }\end{array}$ \\
\hline 1852 & 7166446 & 3145603 & 1848658 & 1310908 \\
\hline 1861 & 9256873 & 4216389 & 2570672 & 1616724 \\
\hline 1871 & 11025786 & 5703944 & 3714304 & 1943182 \\
\hline 1881 & 12625877 & 6410264 & 4147984 & 2207422 \\
\hline 1891 & & & 4064716 & \\
\hline 1901 & 14444175 & 7439941 & 4704396 & 3360665 \\
\hline 1911 & 15613267 & 8162087 & 5480673 & \\
\hline 1921 & 17257012 & 9064650 & 5964154 & 2857685 \\
\hline 1931 & $17304 \quad 164$ & 8994158 & 6079793 & 2600757 \\
\hline 1941 & 18062564 & 9062671 & 6061831 & 2519354 \\
\hline 1951 & 16786405 & 8828968 & 5685516 & 2685217 \\
\hline
\end{tabular}




\begin{tabular}{|c|c|c|c|c|c|c|}
\hline \multicolumn{7}{|c|}{$\begin{array}{c}(A N N E X E I I)-L e s \text { grandes productions (superficie en acres) } \\
\text { QUÉBEC }\end{array}$} \\
\hline Année & Blé & Orge & Avoine & $\begin{array}{l}\text { Pommes } \\
\text { de } \\
\text { terre }\end{array}$ & $\begin{array}{c}\text { Foin } \\
\text { cultivé }\end{array}$ & Sarrasin \\
\hline 1851 & 354886 & 36958 & 495529 & 64762 & - & 46542 \\
\hline 1860 & 213573 & 120677 & 830203 & 105234 & - & 67571 \\
\hline 1870 & 242726 & - & - & 128185 & 1211953 & - \\
\hline 1880 & 224678 & - & - & 123869 & 1495494 & - \\
\hline 1890 & 168929 & 94464 & 1161038 & 122254 & 2178044 & 117739 \\
\hline 1900 & 139826 & 104135 & 1350031 & 127205 & 2548450 & 102673 \\
\hline 1910 & 62882 & 98164 & 1392139 & 123054 & 3231484 & 119466 \\
\hline 1920 & 114303 & 108086 & 1542096 & 146821 & 3657755 & 90254 \\
\hline 1930 & 36833 & 95290 & 1559571 & 132961 & 3806351 & 92675 \\
\hline 1940 & 18121 & 111493 & 1522160 & 148739 & 3800094 & 73227 \\
\hline 1950 & 9839 & 53533 & 1336889 & 93762 & 3516011 & 41938 \\
\hline
\end{tabular}

$$
\begin{gathered}
(A N N E X E I I I)-\text { Les grandes productions végétales } \\
\text { (boisseaux et tonnes) } \\
\text { QUÉBEC }
\end{gathered}
$$

\begin{tabular}{|c|c|c|c|c|c|c|}
\hline Année & $\begin{array}{c}\text { Blé } \\
\text { Boisseaux }\end{array}$ & $\begin{array}{c}\text { Orge } \\
\text { Boisseaux }\end{array}$ & $\begin{array}{c}\text { Avoine } \\
\text { Boisseaux }\end{array}$ & $\begin{array}{c}\text { Pommes } \\
\text { de } \\
\text { terre } \\
\text { Boisseaux }\end{array}$ & $\begin{array}{c}\text { Foin } \\
\text { cultivé } \\
\text { Tonnes }\end{array}$ & $\begin{array}{c}\text { Sarrasin } \\
\text { Boisseaux }\end{array}$ \\
\hline 1851 & 3268875 & 508637 & 9531397 & 4659115 & 755579 & 557879 \\
\hline 1860 & 2798610 & 2423368 & 18568884 & 13460725 & 689977 & 1301404 \\
\hline 1870 & 2058076 & 1668208 & 15116262 & 18068323 & 1225640 & 1676078 \\
\hline 1880 & 2019004 & 1751539 & 19990205 & 14873287 & 1614906 & 2041670 \\
\hline 1890 & 1646882 & $\begin{array}{lll}1580 & 197\end{array}$ & 17818589 & 15861797 & 2243435 & $\begin{array}{llll}2 & 118 & 197\end{array}$ \\
\hline 1900 & 1968203 & 2535597 & 33536677 & $17 \quad 135739$ & 2581823 & 1849596 \\
\hline 1910 & 932459 & 2340364 & 33804291 & 15451539 & 3830628 & 2365539 \\
\hline 1920 & 1576227 & 2320781 & 36838756 & 17745597 & 3258645 & 1500758 \\
\hline 1930 & 554013 & 1947794 & 32860820 & 15200969 & 3843777 & 1486589 \\
\hline 1940 & 270974 & 2505880 & $\begin{array}{lll}35 & 100 & 252\end{array}$ & 22417923 & 3970129 & 1093884 \\
\hline 1950 & 204869 & 1508006 & 36121876 & 16574968 & 3559230 & 859562 \\
\hline
\end{tabular}




\begin{tabular}{|ccccccc|}
\hline \multicolumn{7}{|c|}{ (ANNEXE IV) - Évolution des grands élevages } \\
(en nombre de têtes)
\end{tabular}

\begin{tabular}{|ccccc|}
\hline \multicolumn{1}{|c}{$(\boldsymbol{A N N E X E} \boldsymbol{V})-L a$} & Mauricie dans le Québec, en $\%$ \\
\hline Année & $\begin{array}{c}\text { Superficie } \\
\text { occupée }\end{array}$ & $\begin{array}{c}\text { Superficie } \\
\text { améliorée }\end{array}$ & $\begin{array}{c}\text { Superficie en } \\
\text { gr. cultures }\end{array}$ & $\begin{array}{c}\text { Supericie } \\
\text { en pâturages }\end{array}$ \\
\hline 1852 & 6. & 4.7 & 5. & 4.3 \\
1861 & 5.4 & 4.6 & 5.1 & 3.9 \\
1871 & 5.7 & 4.8 & 5.2 & 4.7 \\
1881 & 6.0 & 5. & 5.6 & 4.8 \\
1891 & - & - & 6.1 & - \\
1901 & 5.8 & 5.8 & 6.0 & 4.8 \\
1911 & 5.8 & 6.3 & 6.3 & -6.2 \\
1921 & 5.6 & 6.3 & 6.5 & 6.3 \\
1931 & 5.3 & 6.2 & 6.2 & 6.3 \\
1941 & 4.9 & 6.1 & 6.1 & 5.6 \\
1951 & 5.0 & 5.7 & 5.9 & \\
\hline
\end{tabular}

Note: Nous indiquons pour 1891 le pourcentage de $6.1 \%$ sous toute réserve. Comme nous ne disposions pas de données nécessaires à la reconstitution de la superficie en culture dans la région, nous avons comparé l'estimation de 1891 pour les trois comtés (281 328 acres après conversion des arpents en acres) à l'estimation proposée par les responsables du recensement de 1941 pour l'ensemble québécois en 1891 (4 610587 acres). Nous croyons que ce pourcentage n'est pas très éloigné de celui que nous aurions obtenu en comparant, avec l'estimation de 1901 les données réelles relatives aux différentes cultures dans la région, si nous avions pu en disposer. 


\begin{tabular}{|c|c|c|c|c|c|c|}
\hline \multicolumn{7}{|c|}{$\begin{aligned}(A N N E X E V I)- & \text { La Mauricie dans le Québec } \\
& \text { superficie des grandes cultures en \% }\end{aligned}$} \\
\hline Année & Blé & Orge & Avoine & $\begin{array}{l}\text { Pommes } \\
\text { de terre }\end{array}$ & $\begin{array}{l}\text { Foin } \\
\text { cultivé }\end{array}$ & Sarrasin \\
\hline 1851 & (4.3) & $(2.5)$ & $(7.1)$ & $(3.9)$ & - & (13.5) \\
\hline 1860 & (5.5) & $(2.4)$ & (6.9) & (4.5) & - & $(9.6)$ \\
\hline 1870 & (5.7) & - & - & (4.8) & $(3.7)$ & - \\
\hline 1880 & (4.9) & - & - & (4.1) & (4.4) & - \\
\hline 1890 & (5.8) & (1.9) & $(7.7)$ & (4.5) & (4.6) & - \\
\hline 1900 & (5.8) & (4.3) & $(7.8)$ & (5.0) & (5.0) & $(9.7)$ \\
\hline 1910 & (5.2) & (4.0) & (7.3) & (5.1) & (5.8) & $(9.9)$ \\
\hline 1920 & (5.0) & (4.4) & (7.4) & (5.3) & (6.3) & $(9.7)$ \\
\hline 1930 & (1.7) & (4.8) & $(6.8)$ & (6.1) & (6.0) & $(8.1)$ \\
\hline 1940 & (1.0) & (3.6) & $(6.6)$ & (5.8) & (6.0) & $(6.9)$ \\
\hline 1950 & (1.0) & (3.3) & $(6.4)$ & (6.7) & $(5.8)$ & $(4.7)$ \\
\hline
\end{tabular}

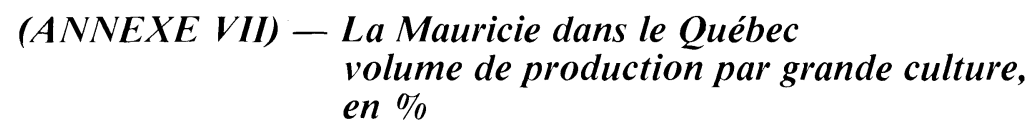

\begin{tabular}{|lcccccc|}
\hline Année & Blé & Orge & Avoine & $\begin{array}{c}\text { Pommes } \\
\text { de terre }\end{array}$ & $\begin{array}{c}\text { Foin } \\
\text { cultivé }\end{array}$ & Sarrasin \\
\hline 1851 & $(5.0)$ & $(2.8)$ & $(8.0)$ & $(4.1)$ & $(6.0)$ & $(10.7)$ \\
1860 & $(5.3)$ & $(2.1)$ & $(7.3)$ & $(4.4)$ & $(6.2)$ & $(8.3)$ \\
1870 & $(3.7)$ & $(1.2)$ & $(8.3)$ & $(4.3)$ & $(4.6)$ & $(6.9)$ \\
1880 & $(4.8)$ & $(2.1)$ & $(8.2)$ & $(3.5)$ & $(6.0)$ & $(10.8)$ \\
1890 & $(5.8)$ & $(1.8)$ & $(7.8)$ & $(4.6)$ & $(5.5)$ & $(10.2)$ \\
1900 & $(5.5)$ & $(3.7)$ & $(7.6)$ & $(4.4)$ & $(5.4)$ & $(8.2)$ \\
1910 & $(5.0)$ & $(3.5)$ & $(6.7)$ & $(4.5)$ & $(5.9)$ & $(8.4)$ \\
1920 & $(4.3)$ & $(3.9)$ & $(6.7)$ & $(4.2)$ & $(7.0)$ & $(7.6)$ \\
1930 & $(1.7)$ & $(4.7)$ & $(6.4)$ & $(6.9)$ & $(5.9)$ & $(6.9)$ \\
1940 & $(1.0)$ & $(3.0)$ & $(5.7)$ & $(5.7)$ & $(5.1)$ & $(5.4)$ \\
1950 & $(1.1)$ & $(3.0)$ & $(6.2)$ & $(7.4)$ & $(5.3)$ & $(3.8)$ \\
& & & & & & \\
\hline
\end{tabular}




\begin{tabular}{|c|c|c|c|c|c|c|c|c|c|c|c|c|}
\hline & & & $(A N N L$ & $E X E V I I$ & I) $-L_{n c}$ & $\begin{array}{l}\text { Maur } \\
\text { mbre }\end{array}$ & $\begin{array}{l}\text { ricie dans } \\
\text { d'animau }\end{array}$ & $\begin{array}{l}\text { le Quél } \\
u x, \text { en } \%\end{array}$ & $\begin{array}{l}\text { bec } \\
b\end{array}$ & & & \\
\hline & & Anı & lée & $\begin{array}{l}\text { Vaches } \\
\text { laitières }\end{array}$ & Ovin & & Pores & Chevaux & Vola & Illes & & \\
\hline & & $\begin{array}{l}18 \\
18 \\
18 \\
18 \\
18 \\
19 \\
19 \\
19 \\
19 \\
19 \\
19\end{array}$ & & $\begin{array}{l}(6.3) \\
(5.3) \\
(5.2) \\
(5.1) \\
(5.4) \\
(5.7) \\
(6.3) \\
(6.1) \\
(6.0) \\
(5.6) \\
(5.8)\end{array}$ & $\begin{array}{l}(6.1) \\
(5.7) \\
(6.1) \\
(6.4) \\
(6.5) \\
(6.6) \\
(5.9) \\
(5.4) \\
(5.5) \\
(5.4) \\
(4.2)\end{array}$ & & $\begin{array}{l}(6.1) \\
(5.5) \\
(5.6) \\
(5.8) \\
(5.9) \\
(6.3) \\
(6.6) \\
(6.8) \\
(6.8) \\
(5.9) \\
(6.5)\end{array}$ & $\begin{array}{l}(4.9) \\
(4.7) \\
(4.7) \\
(4.7) \\
(4.8) \\
(5.1) \\
(5.6) \\
(5.4) \\
(5.4) \\
(5.5) \\
(5.3)\end{array}$ & $\begin{array}{l}(5 . \\
(5 . \\
(5 . \\
(5 . \\
(5 . \\
(5 . \\
(5 .\end{array}$ & & & \\
\hline & NNEXI & $I X)$ & $\begin{array}{l}\text { Les gr } \\
d u \text { tot }\end{array}$ & $\begin{array}{l}\text { andes ca } \\
\text { tal région }\end{array}$ & $\begin{array}{l}\text { atégorie } \\
\text { nal pou }\end{array}$ & $\begin{array}{l}\text { d'occe } \\
\text { certain }\end{array}$ & $\begin{array}{l}\text { upants er } \\
\text { nes année }\end{array}$ & $\begin{array}{l}n \text { Mauric } \\
\text { es. }\end{array}$ & cie, par & $m t$ & en & \\
\hline & & $\begin{array}{l}\text { Saint-Mau } \\
\text { du total } r\end{array}$ & $\begin{array}{l}\text { rice } \\
\text { gaional }\end{array}$ & & $\begin{array}{r}\text { Cha } \\
\% \text { du to }\end{array}$ & $\begin{array}{l}\text { mplain } \\
\text { tal région: }\end{array}$ & & $\%$ du & $\begin{array}{l}\text { Nicolet } \\
\text { total régi }\end{array}$ & & & $\begin{array}{l}\text { pants } \\
\text { gion }\end{array}$ \\
\hline Innée & $\begin{array}{l}\text { Moins de } \\
10 \text { acres }\end{array}$ & $\begin{array}{l}\text { De } 11 \text { à } \\
50 \text { acres }\end{array}$ & $\begin{array}{l}\text { Plus de } \\
50 \text { acres }\end{array}$ & $\begin{array}{l}\text { Moins de } \\
10 \text { acres }\end{array}$ & $\begin{array}{l}\text { De } 11 \text { à } \\
50 \text { acres }\end{array}$ & $\begin{array}{l}\text { Plus de } \\
50 \text { acres }\end{array}$ & $\begin{array}{c}\text { Moins de } \\
10 \text { acres }\end{array}$ & $\begin{array}{l}\text { De } 11 \text { à } \\
50 \text { acres }\end{array}$ & $\begin{array}{l}\text { Plus de } \\
50 \text { acres }\end{array}$ & St-M. & Ch. & Nic. \\
\hline 1852 & 32.8 & 22.8 & 19.0 & 15.0 & 34.4 & 24.5 & 52.1 & 42.6 & 44.9 & 21.6 & 32.9 & 45.3 \\
\hline 1871 & 32.8 & 23.8 & 22.9 & 33.6 & 43.8 & 36.6 & 33.4 & 33.0 & 40.4 & 23.5 & 37.9 & 38.5 \\
\hline 1891 & 29.2 & 30.1 & 17.7 & 31.2 & 40.9 & 39.5 & 39.5 & 28.8 & 42.6 & 22.4 & 37.3 & 40.1 \\
\hline 1911 & 29.0 & 27.1 & 20.7 & 40.2 & 42.6 & 36.8 & 30.7 & 30.2 & 42.3 & 22.9 & 38.1 & 38.9 \\
\hline 1921 & 14.9 & 27.5 & 18.8 & 37.6 & 38.3 & 35.5 & 47.4 & 34.1 & 45.5 & 19.5 & 35.9 & 44.5 \\
\hline 1941 & 41.7 & 30.1 & 19.7 & 18.7 & 42.5 & 34.9 & 39.5 & 27.3 & 45.2 & 21.3 & 35.4 & 43.2 \\
\hline & $\begin{array}{l}\text { Moins de } \\
9 \text { acres }\end{array}$ & $\begin{array}{l}\text { De } 10 \text { à } \\
69 \text { acres }\end{array}$ & $\begin{array}{l}\text { Plus de } \\
69 \text { acres }\end{array}$ & $\begin{array}{l}\text { Moins de } \\
9 \text { acres }\end{array}$ & $\begin{array}{l}\text { De } 10 \text { à } \\
69 \text { acres }\end{array}$ & $\begin{array}{l}\text { Plus de } \\
69 \text { acres }\end{array}$ & $\begin{array}{l}\text { Moins de } \\
9 \text { acres }\end{array}$ & $\begin{array}{l}\text { De } 10 \text { à } \\
69 \text { acres }\end{array}$ & $\begin{array}{l}\text { Plus de } \\
69 \text { acres }\end{array}$ & & & \\
\hline 1951 & 25.0 & 26.5 & 17.6 & 26.1 & 39.0 & 36.4 & 48.8 & 34.4 & 45.8 & 19.0 & 36.7 & 44.1 \\
\hline
\end{tabular}



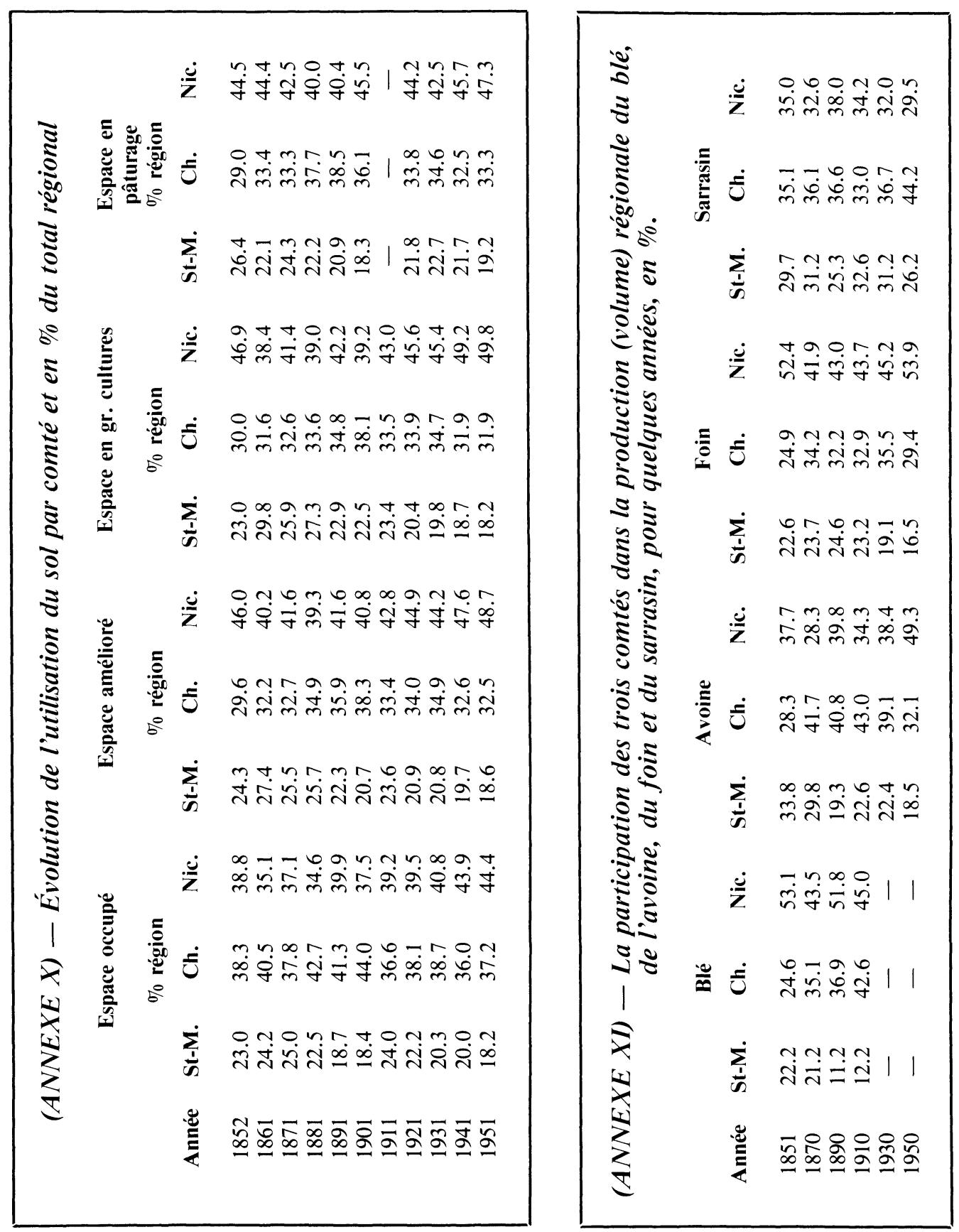


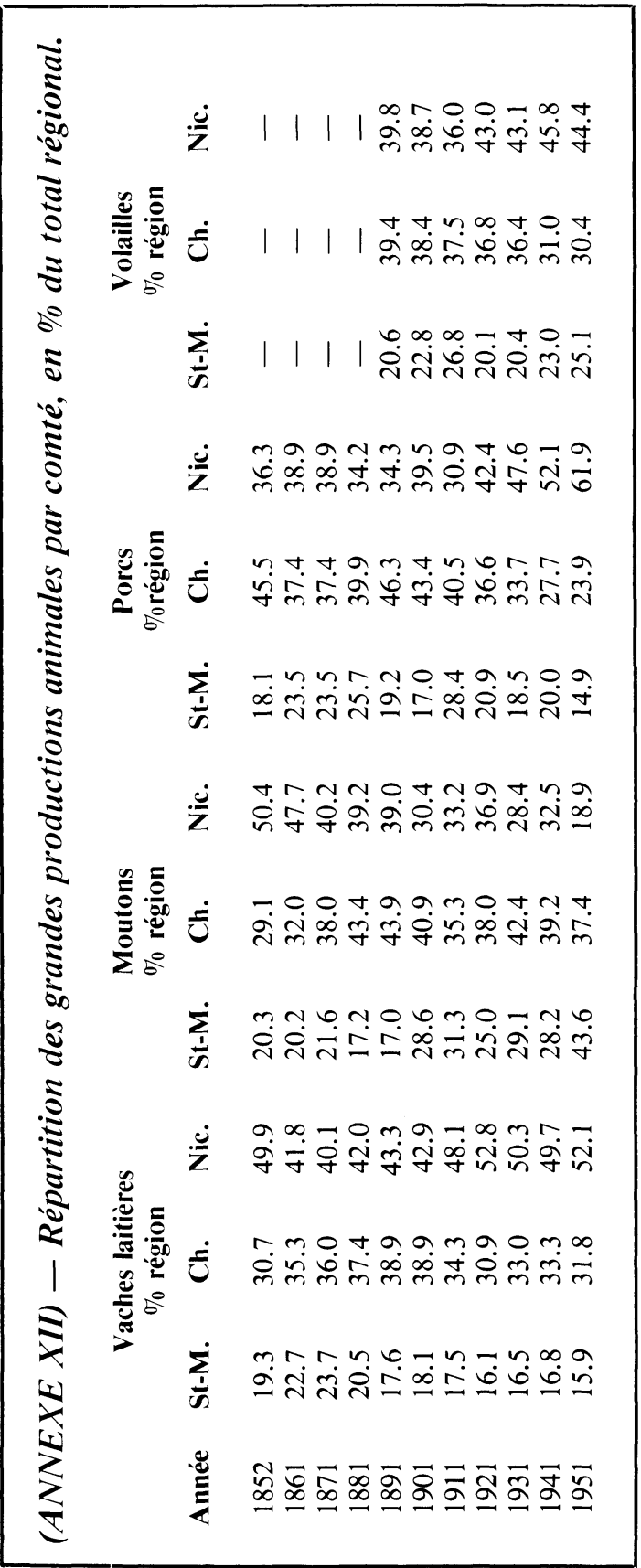

